\title{
Calling on Primary Care to Prevent BRCA-Related Cancers
}

\author{
Katrina Armstrong, $\mathrm{MD}^{1,2}{ }^{\infty}$
}

'Department of Medicine, Massachusetts General Hospital, Boston, MA, USA; ${ }^{2}$ Harvard Medical School, Boston, MA, USA.

With the USPSTF reaffirming the importance of screening, counseling, and testing appropriate women for BRCA1/2 mutations, primary care has both an opportunity and a responsibility to lead in the implementation of these recommendations. Since the last UPSTF recommendations about preventing BRCArelated cancers in 2013, progress in incorporating risk assessment, counseling, and testing into primary care has been slow. There are multiple barriers to implementation outside of primary care, including limitations of the electronic medical record, availability of genetic counseling, and the administrative burden associated with obtaining insurance coverage. However, the early imbalance between hype and evidence in genomics led to a general skepticism among primary care providers about the importance of genomic medicine-a sharp contrast with many other areas of internal medicine. As a growing number of companies offer genetic testing directly to consumers and new models of genetic counseling are developed, primary care should capitalize on the opportunity to lead in the prevention of BRCA-related cancers-both to ensure that these services are delivered appropriately and in coordination with ongoing primary care and that primary care is not left behind as genomic medicine becomes a reality across internal medicine.

$\mathrm{J}$ Gen Intern Med 35(3):903-5

DOI: $10.1007 / \mathrm{s} 11606-019-05469-7$

(C) Society of General Internal Medicine 2019

\begin{abstract}
A little over six years ago, a good friend of mine died of $B R C A 1$-related ovarian cancer. She was a healthconscious mother of three who saw a highly trained primary care doctor on a regular basis. But when she was diagnosed in 2007 , testing for mutations in the breast and ovarian cancer susceptibility genes, $B R C A 1$ and 2, was rare outside of specialized clinics in a few academic centers. There were no BRCA testing USPSTF guidelines, little to no insurance coverage of testing, and limited evidence to guide the use of testing in patients with or without cancer. A lot has changed.

Over the last decade, BRCA testing has moved from a boutique offering to an integral part of breast and ovarian
\end{abstract}

Received September 24, 2019

Revised September 24, 2019

Accepted October 9, 2019

Published online October 21, 2019 cancer risk assessment and breast cancer treatment decisionmaking. The accuracy of testing has improved with new sequencing platforms and the ability to measure genomic rearrangements. Extensive evidence now links test results to clinical outcomes, even down to mutation specific cancer risks. Women found to carry mutations have multiple options for breast and ovarian cancer risk reduction, including prophylactic surgery, which has been shown reduce cancer risk by at least $80 \%$. Over a dozen companies now offer testing for mutations in BRCA1 and 2, greatly reducing the cost of testing.

Given these developments, it is great to see that the US Preventive Services Taskforce has reaffirmed their recommendation about risk assessment and genetic counseling and testing for BRCA-related cancers. ${ }^{1}$ In 2013 , the USPSTF recommended that women with a family history of breast, ovarian, tubal, or peritoneal cancers be screened with a brief tool to assess their risk of carrying a $B R C A 1$ or 2 mutation and, if they meet risk criteria, undergo genetic counseling and, if indicated, BRCA testing. The 2019 recommendations expand that recommendation to include patients with a personal history of one of these cancers or with ancestry associated with increased risk of carrying a mutation, primarily Ashkenazi Jewish and French-Canadian in the USA. These recommendations are consistent with those of the American Society of Clinical Oncology, the American College of Obstetrics and Gynecology, and the National Comprehensive Cancer Network..$^{2-4}$

Although USPSTF recommendations to offer BRCA testing to appropriate women are now six years old, implementation has been slow for several reasons. Family history information is incomplete in most electronic medical records (EMR), making it difficult to create decision support or population registries to identify patients who should be screened. EMR data on ancestry associated with BRCA mutations is likely to be even less common. Most of the screening tools recommended by the USPTSF are relatively complicated and cumbersome to integrate into provider workflow or patient questionnaires. Genetic counseling resources are limited and rarely included in the testing services provided by online companies. Insurance coverage is inadequate, particularly in Medicaid and individual market plans. Although insurance companies are required to cover genetic counseling and testing for women who meet eligibility criteria under the affordable care act, obtaining payment often requires extensive approval processes. 
Challenges to implementation continue even after testing has been completed. Although the USPSTF guidelines focus on BRCA testing, most companies now offer a breast cancer gene panel test and most patients will end up undergoing testing for multiple breast cancer susceptibility genes in addition to $B R C A 1$ and 2. For many of those genes, relatively little is known to guide decision-making and the identification of a variant creates both current uncertainty about risk reduction options and the need to reevaluate the test results over time as new evidence becomes available. ${ }^{5}$ Given that sequencing and variant calling methodologies vary across companies, results may also vary across companies and the accuracy of a particular test is difficult to judge. ${ }^{6}$ Currently, few electronic medical records (EMRs) incorporate genetic test results in any format other than a scanned document, making it hard to retrieve results efficiently or to incorporate results into decision support. ${ }^{7}$ Furthermore, access to specialty cancer genetic programs to assist with follow-up of women found to carry mutations is limited in many areas.

Although these challenges are real and important, the reality is that the greatest challenge to the implementation of these recommendations has been insufficient engagement of the primary care community. The admirable tendency of primary care providers to see through hype and search for evidence before adopting a new technology led to healthy skepticism about the potential benefit of genomics during the initial decade of that BRCA testing was clinically available. Most studies suggested that while the majority of primary care providers were aware of BRCA testing, only a minority had ordered it. ${ }^{8,9}$ This situation was probably exacerbated by the initial price of BRACA testing ( $\sim 2400)$, which was hard to align with the growing commitment to high value care in the primary care community. The complexity of early BRCA testing guidelines no doubt contributed to the relatively low levels of knowledge and high levels of discomfort with selecting appropriate patients for testing. Furthermore, there has been relatively little interest in clinical or translational genomic research in the general internal medicine research community. From the over 9300 articles published in the Journal of General Internal Medicine since 2002, only 20 include the word genetic or genomic. In the over 20 years that I have spent mentoring fellows and junior faculty in academic general internal medicine, I have met only two general internal medicine physician scientists committed to studying genomic medicine.

Given these new USPSTF recommendations, it is increasingly clear that it is time for primary care leadership to put aside this initial skepticism and assume responsibility for overcoming these implementation challenges. The accompanying evidence review provides convincing data that appropriate use of BRCA testing saves lives, which satisfied the rigorous USPSTF recommendation and should satisfy even the most evidence-based primary care provider. ${ }^{10}$ Although overcoming these challenges will not be simple, there are several positive developments that can and should be harnessed for these next steps. EMR integration of genetic test results is advancing through the creation of FHIR standards with genomic modules, the eMERGE program at the National Human Genome Research Institute Rand the attention of large EMR companies. ${ }^{11,12}$ New models of telehealth genetic counseling have been developed to support both patient and provider consultations. ${ }^{13}$ Population health efforts are increasingly recognizing the importance of collecting information on family history. The price of testing has dropped to around $\$ 200$ as new companies have entered the market. For these and other reasons, virtually every medical subspecialty is now embracing the opportunity to implement clinical genomics to improve patient outcomes - even in situations where the evidence of benefit is far less convincing than BRCA testing.

In June of this year, my friend's youngest child spoke at his high school graduation ceremony. Sitting in the audience, I knew how proud she would be. I also know how proud she would be to see the progress that has been made towards preventing women from dying from BRCA-related cancers, including the latest USPSTF recommendations. Now, it is time for primary care to assume the responsibility for implementing those recommendations so that women with BRCAl and 2 mutations have an opportunity to learn about their risk before they learn they have been diagnosed with cancer.

Corresponding Author: Katrina Armstrong, MD; Department of Medicine Massachusetts General Hospital, Boston, MA, USA (e-mail: karmstrong6@mgh.harvard.edu).

\section{Compliance with Ethical Standards:}

Conflict of Interest: The author declares that she does not have a conflict of interest.

\section{REFERENCES}

1. USPSTF. Risk assessment, genetic counseling, and genetic testing for BRCA-related cancer. JAMA 2019;322:652-65.

2. Robson ME, Bradbury AR, Arun B, et al. American Society of Clinical Oncology policy statement update: genetic and genomic testing for cancer susceptibility. J Clin Oncol 2015;33:3660-7.

3. Committee opinion no. 634: Hereditary cancer syndromes and risk assessment. Obstet Gynecol 2015;125:1538-43.

4. Genetic/familial high-risk assessment: breast and ovarian. national comprehensive cancer network; 2019.

5. Easton DF, Pharoah PD, Antoniou AC, et al. Gene-panel sequencing and the prediction of breast-cancer risk. N Engl J Med 2015;372:224357.

6. Amendola LM, Jarvik GP, Leo MC, et al. Performance of ACMG-AMP variant-interpretation guidelines among nine laboratories in the clinical sequencing exploratory research consortium. Am J Hum Genet 2016;99:247.

7. Green ED, Guyer MS, National Human Genome Research I. Charting a course for genomic medicine from base pairs to bedside. Nature 2011;470:204-13. 
8. Burke W, Culver J, Pinsky L, et al. Genetic assessment of breast cancer risk in primary care practice. Am J Med Genet A 2009;149A:349-56.

9. Guerra CE, Sherman M, Armstrong $\mathbf{K}$. Diffusion of breast cancer risk assessment in primary care. J Am Board Fam Med 2009;22:272-9.

10. Nelson HPM, Cantor A, Haney E, Holmes R. Risk assessment, genetic counseling, and genetic testing for BRCA-related cancer in women. Updated Evidence Report and Systematic Review for the US Preventive Services Task Force. JAMA;322:666-85.

11. Aronson S, Babb L, Ames D, et al. Empowering genomic medicine by establishing critical sequencing result data flows: the eMERGE example. J Am Med Inform Assoc 2018;25:1375-81.
12. Williams MS, Kern MS, Lerch VR, Billet J, Williams JL, Moore GJ. Implementation of a patient-facing genomic test report in the electronic health record using a web-application interface. BMC Med Inform Decis Mak 2018;18:32.

13. Zierhut HA, MacFarlane IM, Ahmed Z, Davies J. Genetic counselors' experiences and interest in telegenetics and remote counseling. J Genet Couns 2018;27:329-38.

Publisher's Note Springer Nature remains neutral with regard to jurisdictional claims in published maps and institutional affiliations. 